\title{
How Australia deals with suicides: Lessons learnt and implications for Sri Lanka
}

\author{
Amila Chandrasiri \\ School of Population and Global Health, University of Melbourne, Australia. \\ Correspondence: Dr. P.A.Amila Chandrasiri \\ e-mail: amilachan@yahoo.com \\ https://orcid.org/0000-0002-3242-1489 \\ Submitted on 06.01.2021 and accepted for publication on 27.03.2021
}

\section{Introduction}

Suicide has emerged as a serious public health issue worldwide, with nearly 800,000 people succumbing to suicide every year (1). Suicides stood as the $16^{\text {th }}$ leading cause of death globally while accounting for $1.4 \%$ of all deaths worldwide by 2016 (2). Sri Lanka is also experiencing the 'suicide epidemic' as nearly 4,000 deaths are reported each year. There were 4523 deaths by suicide in 2017 and the mortality rate was reported as 19.8 per 100000 people (3). It is estimated that for each adult who dies by suicide, twenty more may have made an attempt. Suicides add a significant financial and social burden as well (4).

Several initiatives were taken by the government and health authorities to address this issue. Some of these strategies brought intended results as a decline in the overall suicide rates in Sri Lanka has been noted from 60 per 100,000 in 1995 to 18.5 per 100,000 people by 2011 (5). Recommendations suggested in the 1997 report of the Presidential Committee on the prevention of suicides were remarkable milestone in suicide prevention in Sri Lanka. Regulatory controls on the importation and sales of highly toxic pesticides were the most important contributing factor to this drop (6).

However, still suicides stand as a significant health issue in Sri Lanka. Therefore, recent changes in the social context of Sri Lanka like urbanisation, rise of information technology and substance use should be carefully addressed in managing this problem (5). Experiences and lessons from developed countries will provide a vital insight for designing and implementing prevention strategies.
Australia has been paying top priority for suicide prevention strategies over the last decade. Key priority has been extended and evidence-based interventions were implemented with a strategic vision (7). Promoting help seeking behaviour, active involvement of primary care, multi stakeholder involvement and reducing stigma can be identified as key factors behind the successful implementation of these preventive strategies (7). These interventions make several implications for the Sri Lankan context. This review is aimed at critically analysing the suicide prevention strategies in Australia and identifying implications for Sri Lanka.

\section{Suicide epidemiology in Australia}

More than 3000 Australians end their lives each year and it accounts for 8 deaths per day. There were 3,046 suicide deaths in 2018. Three fourths of these deaths are among males. Young suicides is another pressing issue in Australia as suicide ranks as a leading cause of death for Australians between the ages of 15 and 24 years while accounting for $36 \%$ of deaths in the particular age group. The suicide rate among Indigenous Australians is twice that of their nonIndigenous counterparts (8).

\section{Policy directives and legislative support}

Australia is governed under a Federal System and significant authority is vested on State Governments. However, the Federal Government provides important policy directives for key national health 
issues. But State Governments enjoy the freedom to design and implement own actions based on national strategies (9).

The National Suicide Prevention Strategy (NSPS) provides the platform for Australia's national policy on suicide prevention with an emphasis on promotion, prevention and early intervention. The National Government declared a renewed approach to suicide prevention in November 2015 based on the recommendations of National Mental Health Commission Review (10). A new National Suicide Prevention Strategy was drafted and enacted.

To further strengthen the national response, The Fifth National Mental Health and Suicide Prevention Plan, which is popularly known as the Fifth Plan was endorsed by the National Government in August 2017 (11). This has reiterated the commitment from all State Governments to work together to achieve integrated planning and service delivery of mental health for suicide prevention.

\section{Main aims}

The suicide prevention strategy in Australia is based on three main aims; (i) raising awareness about suicide risk in the community, (ii) helping those at risk of suicide and (iii) supporting affected individuals including those who have lost someone close (10).

These aims provide a holistic guidance for the suicide prevention activities in Australia.

\section{Key strategies}

\section{1 - Mental Health First Aid (MHFA) programme}

The Mental Health First Aid (MHFA) programme was developed and implemented in Australia with the objective of training community members to help someone who is undergoing a mental health crisis situation that would lead to suicide (12). It has been showing promising results in reducing suicides (13). Because it enables the detection of problems early and encourage people at risk to seek help. So MHFA is particularly useful in suicide prevention. Over the last decade, MHFA has been successfully introduced to several developed and developing countries (13).

\section{2 - Paying more attention on vulnerable groups}

Identification of vulnerable groups is of utmost importance in planning public health strategies to prevent attempted suicides (14). A special Aboriginal and Torres Strait Islander Suicide Prevention Strategy was implemented focusing Indigenous Australians, considering the higher suicide rates among them (8). Youth is another key target group in the Australian suicide response. Youth suicide rates are known to be lower in areas with targeted suicide prevention activities in Australia (15).

\section{3 - More responsibilities were assigned to Primary Health Networks (PHNs)}

This was a key initiative to promote the involvement of primary care system for suicide prevention. Primary Health Networks (PHNs) have been tasked with commissioning regionally appropriate suicide prevention activities and services since July 2016 (10). PHNs were encouraged to work closely with local organizations to reach people who are at the risk of suicide. This approach focuses on effective local coordination and management, and allows for community needs and strengths to be recognised. Special training was given to General Practitioners (GP) to deliver support and preventive services. Delivering culturally appropriate care is a key principle at primary care level. Linking suicide prevention services with existing primary health care system is known to be effective(14).

\section{4 - Multi stakeholder involvement}

Multi stakeholder involvement is a key feature in national response against suicides in Australia (11). Several government institutes and nongovernmental organisations work closely to a common agenda. Universities and research institutes make a crucial contribution by generating research evidence in order to guide actions. Australia follows a multilevel systems approach. This approach requires that components ranging from individual-level to public health interventions are implemented simultaneously in a localised region. 


\section{5 - Anti-stigma and awareness campaigns}

Suicide and stigma are related in a two way direction in which suicide can cause stigma and stigma can also lead to suicidal thoughts (16). On the other hand, stigma has been identified as a major barrier in accessing services and hence prime attention has been focused on anti-stigma strategies. In order to ensure more people are accessing the help they require, mental health service providers should create a stigma free environment (17). The main objective of the anti-stigma and awareness campaigns was to promote help seeking behaviour of people in Australia.

\section{6 - Crisis support services and help lines}

Australia has a well-organised phone-based crisis support services which can be accessible $24 \times 7$. It provides crisis support services for anyone who experience a personal crisis or have suicidal thoughts. Lifeline is the main crisis support services which has a high utilisation. It consists of a national infrastructure with 40 centres and 4,500 crisis supporters (18). High utilisation is a factor to show the success of this service as every 30 seconds, a person in Australia reaches out to Lifeline for help. Assurance of confidentiality, professionalism shown by the supporters and $24 \times 7$ accessibility are the key factors contributing to its success. Because clients perceive that the 'Lifeline' listen to them without judgment and work together to explore options for the problem. On the other hand, general help seeking behaviour of the community promotes high utilisation. Because there is a general perception in the society that, "it is OK to have suicidal feelings and it is important to reach out and let someone know how you're feeling" (18).

\section{7 - Supporting affected individuals including those who have lost someone close}

Survivors of suicidal attempts and affected family members go through the complicated process of grief which is specifically characterised by the felling of guilt, shame, denial and anger (19). The grieving process, more often than in other causes of death, doesn't integrate but is complicated with prolonged grief. This is key aspect of suicide prevention strategy in Australia.
Several interventions have been implemented to help families and loved ones of those who committed or attempted suicide. Because they also undergo an immense psychological trauma as a consequence. So it is important to address their psychological well-being.

\section{8- Guiding responsible media reporting}

Appropriate reporting of suicides in the media plays a crucial role in preventing suicide and increasing public understanding. Because inappropriate reporting can alienate members of the community, sensationalise the issue or inadvertently glamorize suicide. Royal Australian and New Zealand College of Psychiatrists (RANZCP) has introduced a comprehensive set of guidelines to advocate and guide media in responsible reporting of suicides (20).

\section{Implications for Sri Lanka}

\section{1 - Strong and regular policy directive}

Sri Lanka has been paying more attention on suicide prevention through policy and regulatory approaches (6). But like in Australia, a continuous commitment is needed. It is important to regularly update the national strategies. Obtaining active involvement of Universities is also important to ensure evidence-based policy making. Universities can contribute by offering research-based guidance. More responsibilities can be assigned to provincial and district health authorities in Sri Lanka.

\section{2 - Promoting help seeking}

A massive social marketing is warranted to promote the availability and accessibility to help services in Sri Lanka. Sri Lanka has suicide helplines. Though the facility delivers a commendable service, a gap exists between the demand and utilisation. Chandrasiri et al., (2019), reported that only 5\% of patients who admitted to hospitals following deliberate self-harm, contacted the helpline before the act (21). This indicates a huge gap. Lack of awareness and fear of stigma can be identified as main reasons behind. So exploring how Australia made such help services more usable will provide 
important implications for Sri Lanka. There are posters and infographs about the importance of seeking help, when to seek help and how to seek help. They are displayed in public places in Australia and not restricted to health care settings.

\section{3 - Culturally appropriate services}

Cultural awareness and cultural sensitivity are two of the key attributes in Australian suicide prevention campaign. As most of the predisposing factors for suicides and self-harm are embedded with sociocultural context of the person, showing cultural awareness is crucial while having a generic approach (22). Defining vulnerable groups and approaching them through culturally sensitive interventions is also important. Sri Lanka is showing a unique trends in suicide epidemiology as young and female suicides are rising (23). Therefore, targeted interventions which are designed in culturally appropriate manner is highly recommended.

\section{4 - Delivering 'settings approach' for suicide prevention}

Australia is promoting settings approach for suicide prevention as school and work place based programmes have been designed (7). This will be a good example for Sri Lanka as well. Because Sri Lanka has a well-organized school health system, it can be successfully utilised to deliver such programmes. Rising numbers of deliberate self-harm among adolescents is a known problem in Sri Lanka (23).

\section{5 - Involvement of primary care services}

As GPs play a key role in suicide prevention activities, Sri Lanka can also use the potential of primary health care system to deliver suicide prevention services. It is good to see that under the ongoing Primary Care Strengthening Project (PSSP) more attention has been paid on improving mental health services in primary care level. So, policy makers and health care planners can incorporate suicide prevention interventions to the PSSP package. As Sri Lanka doesn't have an organised GP system at the moment, 'family doctors' (who is synonymous with GPs in Sri Lanka) who deliver outpatient care in private sector dispensaries can be empowered to deliver mental health services at primary care level. Sensitization and training should be done to enhance knowledge and skills of primary care work force. Gaps in the psychiatric care received by the patients who are admitted following DSH has been reported as only one third of such patients undergo psychiatric assessment in hospitals (21). Community-based outreach programmes will be another effective strategy to improve suicide prevention services provided for patients with psychiatric illnesses. Competencies on preliminary risk assessments and providing basic psychological support should be improved among primary care workforce including doctors and nurses (14).

\section{6 - Promoting online services for awareness and prevention}

Most of suicide prevention services in Australia interact with public and deliver their services online. This has several advantages like quick access and perception of confidentiality (24). This can be promoted in Sri Lanka as well. Lower IT literacy and poor internet access may arise as barriers. But the current policy directions by the government to promote IT use among general public provides a favourable situation.

\section{7 - Reducing stigma}

Anti-stigma interventions should be actively delivered to every suicide survivor (25). Mental health professionals free of stereotyping and should not be judgmental. More training opportunities on psychiatric management DSH patients should be given for doctors in curative sector (14). This issue should be incorporated into basic training of health professionals and more in-service training should also be arranged. This will ensure better accessibility to mental health services by public. Reducing stigma will also be helpful in improving social integration of survivors after the attempt (25). 


\section{8 - Advocating and providing guidance for responsible media reporting}

Both availability of reporting and assurance of adhering to respective guidelines are important. Media professionals and health experts should work collaboratively to balance newsworthiness against the risk of encouraging modeled suicidal behaviour when reporting such incidents (20). Having a regulatory system is important with continuous surveillance.

\section{9 - Introducing Mental Health First Aid (MHFA) programme}

Implementing MHFA training in Sri Lanka will be helpful in supporting people with suicidal ideation (12). Because MHFA had shown successful outcome in several lower and middle income countries (13). Experience from Australia will be really helpful in identifying potential target groups for training. In the Sri Lankan context, primary health care workers like public health midwives and public health inspectors can be trained first. Then they can serve as trainers for community members.

\section{Conclusions}

Suicide has emerged as a serious public health issue worldwide and Sri Lanka is also experiencing the 'suicide epidemic' as nearly 4,000 deaths are reported each year. Despite several efforts to control, suicides continue to stand as a significant health issue. Experiences and lessons from developed countries who had succeeded in suicide prevention will provide a vital insight in designing and implementing prevention strategies. So this review was done with the objective of critically analysing the suicide prevention strategies in Australia and identifying implications for Sri Lanka.

The National Suicide Prevention Strategy (NSPS) provides the platform for Australia's national policy on suicide prevention. To strengthen the national response further, the Fifth National Mental Health and Suicide Prevention Plan, was endorsed by the National Government in August 2017. Mental Health First Aid (MHFA) programme paying more attention on vulnerable groups, assigning more responsibilities to Primary Health Networks (PHNs), multi stakeholder involvement, anti-stigma and awareness campaigns, crisis support services and helplines, support affected individuals including those who have lost someone close and guiding responsible media reporting can be identified as successful strategies implemented in Australia to cope with the suicide.

Based on these practices, Sri Lanka can learn many lessons to strengthen the national response against suicides. A strong and regular policy directive should be made while assigning more responsibilities to provincial and district health authorities. Promoting a help seeking a social marketing campaign is also warranted. Ensuring cultural appropriateness of services delivered is important and delivering 'settings approach' for suicide prevention should be tried. Involvement of primary care services and community-based outreach programme will also be useful. Introducing measures to reduce stigma and promoting online services for awareness are equally important. Initiating Mental Health First Aid (MHFA) programme is another effective strategy by which many of who are at risk would benefit. Last but not the least, advocating and providing guidance for responsible media reporting should follow all the above activities.

\section{References}

1. World Health Organization. Suicide Data 2020. Available from: https://www.who.int/mental_health/prevention/ suicide/suicideprevent/en/

2. World Health Organization. World Health Statistics Overview 2019.

3. IfH M. Evaluation. Global Burden of Disease Collaborative Network. Global Burden of Disease Study 2016 (GBD 2016) results. Institute for Health Metrics and Evaluation Seattle. 2017.

4. Czernin S, Vogel M, Flückiger M, Muheim F, Bourgnon J-C, Reichelt M, et al. Cost of attempted suicide: a retrospective study of extent and associated factors. Swiss Medical Weekly. 2012; 142: w13648.

5. Knipe DW, Padmanathan P, Muthuwatta L, Metcalfe C, Gunnell D. Regional variation in suicide rates in Sri Lanka between 1955 and 2011: a spatial and temporal analysis. BMC Public Health. 2017; 17(1): 193 
6. Knipe DW, Chang S-S, Dawson A, Eddleston M, Konradsen F, Metcalfe C, et al. Suicide prevention through means restriction: Impact of the 2008-2011 pesticide restrictions on suicide in Sri Lanka. PloS one 2017; 12(3): e0172893.

7. Krysinska K, Batterham PJ, Tye M, Shand F, Calear AL, Cockayne $\mathrm{N}$, et al. Best strategies for reducing the suicide rate in Australia. Australian \& New Zealand Journal of Psychiatry. 2016; 50(2): 115-8.

8. Elliott-Farrelly T. Australian Aboriginal suicide: The need for an Aboriginal suicidology? Australian e-journal for the Advancement of Mental Health. 2004; 3(3): 138-45.

9. Duckett S, Willcox S. The Australian health care system: Oxford University Press; 2015

10. Australian Government. National suicide prevention strategy 2016. Available from: https://www1.health.gov.au /internet/main/publishing.nsf/Content/mental-nsps

11. Australian Government. Fifth National Mental Health and Suicide Prevention Plan 2019. [cited 2019 December 2 $\left.2^{\text {nd }} 2020\right]$. Available from: https://www.mentalhealthcommission.gov.au/Monitoringand-Reporting/Fifth-Plan/5th-National-Mental-Healthand-Suicide-Prevention.

12. Jorm AF, Kitchener BA, Reavley NJ. Mental Health First Aid training: lessons learned from the global spread of a community education programme. World Psychiatry. 2019; 18(2): 142-143.

13. Jorm AF, Kitchener BA. Noting a landmark achievement: mental health first aid training reaches $1 \%$ of Australian adults. Taylor \& Francis; 2011.

14. Chandrasiri A, Gunethunga W, Rajapaksha H. Managing deliberate self harm patients: knowledge and practices among hospital doctors in Galle district. Journal of the Ruhunu Clinical Society. 2018;23(1): 16-21.

15. Page A, Taylor R, Gunnell D, Carter G, Morrell S, Martin G. Effectiveness of Australian youth suicide prevention initiatives. The British Journal of Psychiatry. 2011; 199(5): 423-9.
16. Kučukalić S, Kučukalić A. Stigma and Suicide. Psychiatr Danub. 2017; 29(Suppl 5): 895-9.

17. Dalky HF. Mental illness stigma reduction interventions: Review of intervention trials. Western Journal of Nursing Research. 2012;34(4): 520-47.

18. Lifeline. Lifeline 2020. Available from: https://www.lifeline.org.au/?gclid=EAIaIQobChMIubK6 w-n67QIVk3wrCh37rAa8EAAYASAAEgIc1vD BwE

19. Cvinar JG. Do suicide survivors suffer social stigma: a review of the literature. Perspectives in Psychiatric Care. 2005; 41(1): 14-21.

20. RANZCP. Suicide reporting in the media 2019. Available from: https://www.ranzcp.org/news-policy/policy-andadvocacy/position-statements/suicide-reporting-in-themedia.

21. Chandrasiri P, Gunethunga W, Rajapakshe H. Perception on Psychiatric Care Received by Patients Admitted Following Deliberate Self Harm to Three Hospitals in Galle District. International Journal of Multidisciplinary Studies. 2019; 6(1): 48-55

22. Goldston DB, Molock SD, Whitbeck LB, Murakami JL, Zayas LH, Hall GCN. Cultural considerations in adolescent suicide prevention and psychosocial treatment. American Psychologist. 2008; 63(1): 14.

23. Chandrasiri P, Lokubalasuriya A, Hettiarachchi R. Epidemiology of attempted suicides in Southern Sri Lanka. Galle Medical Journal. 2018; 23(1): 7-14.

24. Jacob N, Scourfield J, Evans R. Suicide prevention via the internet. Crisis. 2014.

25. Scocco P, Preti A, Totaro S, Corrigan PW, Castriotta C. Stigma, grief and depressive symptoms in help-seeking people bereaved through suicide. J Affect Disord. 2019; 244: 223-30. 